\title{
Free Magnetic Energy Changes in AR 8210
}

\author{
Brian T. Welsch ${ }^{1}$ and George H. Fisher ${ }^{1}$ \\ ${ }^{1}$ Space Sciences Laboratory, University of California, 7 Gauss Way, Berkeley, CA 94720-7450
}

\begin{abstract}
Fourier local correlation tracking (FLCT) was applied to time series of the normal magnetic field in vector magnetograms of NOAA AR 8210, from 17:13-21:29 UT on 1998 May 1, to derive photospheric flows. These flows, combined with both the observed horizontal magnetic field and an inferred horizontal potential magnetic field, can be used to derive the flux of free magnetic energy across the photosphere. Here, we present the formalism used, as well as a map of the time-averaged free energy flux. Over the time interval covered by the magnetograms, $\sim 10^{31}$ erg of free magnetic energy flowed upward across the photosphere, a significant fraction of the energy typically released in large flares coronal mass ejections (CMEs). A flare and coronal mass ejection began at 22:30 UT. We acknowledge the kind support of AFOSR's MURI program.
\end{abstract}

Keywords. Sun: magnetic fields, Sun: photosphere, Sun: coronal mass ejections (CMEs), Sun: flares, MHD

Assuming ideal MHD describes photospheric magnetic field evolution, the Poynting flux of magnetic energy across the photosphere, $S_{n}$, is a function of the plasma velocity $\mathbf{v}$ and magnetic field $\mathbf{B}$,

$$
S_{n}=\mathbf{B}_{h} \cdot\left(v_{n} \mathbf{B}_{h}-\mathbf{v}_{h} B_{n}\right) / 4 \pi,
$$

where the subscripts $n$ and $h$ refer to normal and horizontal vector components, respectively. Welsch (2006) showed that energy changes in the potential magnetic field, $\mathbf{B}^{(P)}$, that matches the normal component $B_{n}$ of the actual magnetic field $\mathbf{B}$ can also be expressed as a flux,

$$
S_{n}^{(P)}=\mathbf{B}_{h}^{(P)} \cdot\left(v_{n} \mathbf{B}_{h}-\mathbf{v}_{h} B_{n}\right) / 4 \pi .
$$

The free magnetic energy, $U_{M}^{(F)}$, present in the corona is the difference between the magnetic energy $U_{M}$ of the actual field, $\mathbf{B}$, and the magnetic energy $U_{M}^{(P)}$ of the potential field, $\mathbf{B}^{(P)}$, integrated over the coronal volume,

$$
U_{M}^{(F)}=U_{M}-U_{M}^{(P)}=(8 \pi)^{-1} \int d V\left(\mathbf{B} \cdot \mathbf{B}-\mathbf{B}^{(P)} \cdot \mathbf{B}^{(P)}\right) .
$$

Since $\mathbf{B}^{(P)}$ has the minimum possible energy among the set of all magnetic fields that match the observed $B_{n}$, and $B_{n}$ is thought to be unchanged by flares and CMEs, $U_{M}^{(F)}$ quantifies the energy available in $\mathbf{B}$ that can be released during flares and CMEs.

In analogy with $U_{M}^{(F)}$, the flux of free magnetic energy across the photosphere, $S_{n}^{(F)}$, is the difference between the fluxes of enery in the actual and potential fields,

$$
S_{n}^{(F)}=S_{n}-S_{n}^{(P)}=\left(\mathbf{B}_{h}-\mathbf{B}_{h}^{(P)}\right) \cdot\left(v_{n} \mathbf{B}_{h}-\mathbf{v}_{h} B_{n}\right) / 4 \pi .
$$

Démoulin and Berger (2003) suggested that $B_{n} \mathbf{u}^{(L C T)}=-\left(v_{n} \mathbf{B}_{h}-\mathbf{v}_{h} B_{n}\right)$, where $\mathbf{u}^{(L C T)}$ is the flux transport velocity determined by local correlation tracking. (Schuck [2005], however, argued that this equivalence is only approximate.) Using $\mathbf{u}^{(L C T)}$ derived by FLCT (Welsch et al. 2004) with a Gaussian apodization window of 15 pixels applied to vector magnetograms of AR 8210, at 17:13 and 21:29 UT on 1998 May 1, we computed $S_{n}^{(F)}$, shown in grayscale in Figure 1. Flows were only derived in regions with $\left|B_{n}\right|>$ 


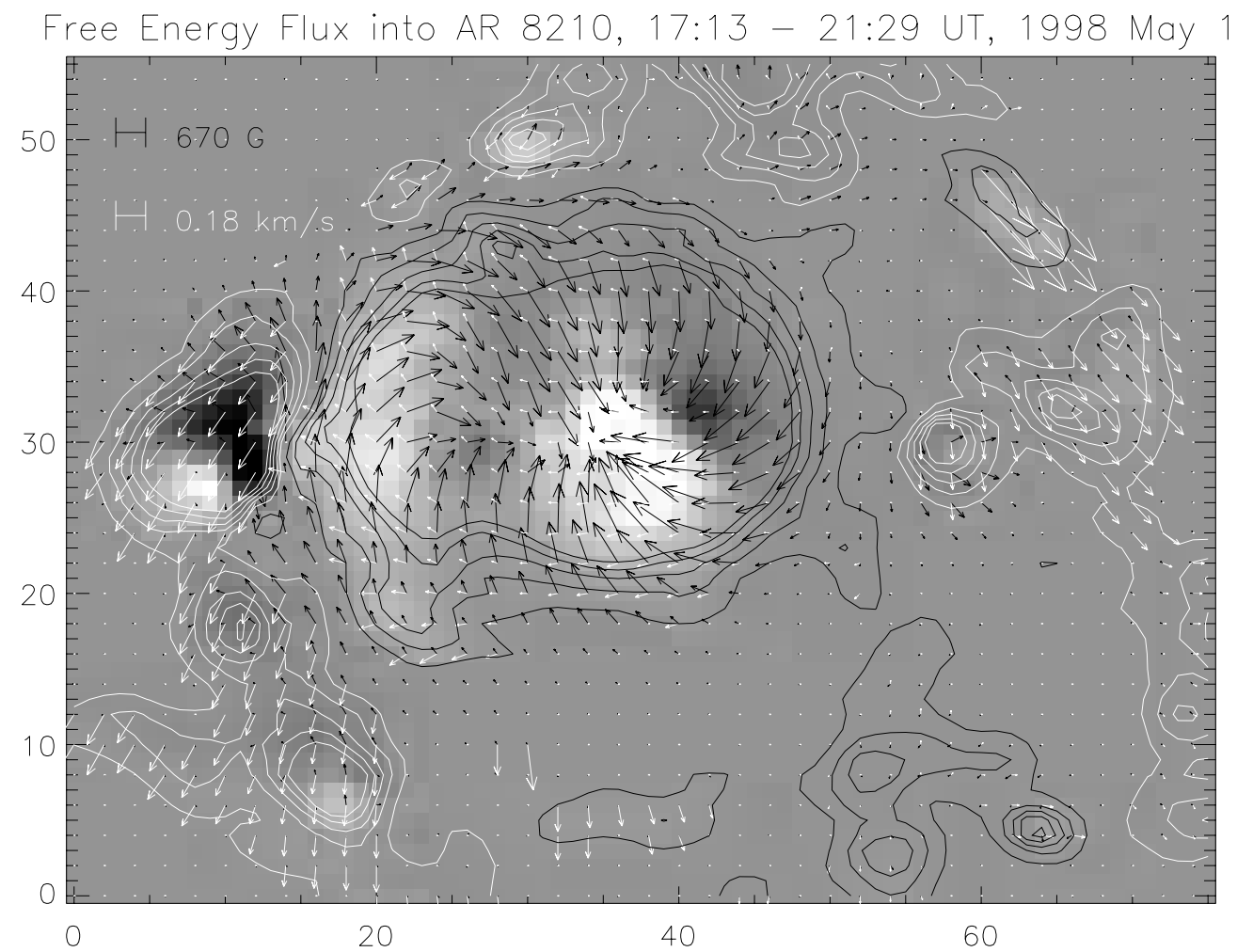

Figure 1. Grayscale image of $S_{n}^{(F)}$, the flux of free energy across the photosphere; white \& black correspond to upward \& downward (resp.) fluxes. Black vectors are the measured $\mathbf{B}_{h}$, and white vectors are derived flows, $\mathbf{u}^{(L C T)}$. Black \& white contours are $100 \mathrm{G}$ level curves of negative \& positive (resp.) regions of $B_{n}$. (For clarity, only contours up to $500 \mathrm{G}$ in $\left|B_{n}\right|$ are shown.)

$100 \mathrm{G}$. Over the $4 \mathrm{hr}$., $16 \mathrm{~min}$. time interval, $\Delta t$, covered by the magnetograms, $\Delta U_{M}^{(F)}=$ $\Delta t\left(\int d a S_{n}^{(F)}\right) \simeq 10^{31}$ erg of free magnetic energy flowed upward across the photosphere, a significant fraction of the energy released in large flares and CMEs. A flare and CME began at 22:30 UT.

Currently, few time sequences of high-quality vector magnetograms exist around the times of flares and CMEs, but future magnetographs (e.g., SOLIS, FPP on Solar-B, and HMI on SDO) should enable quantitative, statistical studies of relationships between the flux of magnetic free energy and energy release processes in the corona.

\section{References}

Démoulin, P. and Berger, M. A. 2003, Sol. Phys. 215, 203

Schuck, P. 2005, ApJ 632, L53

Welsch, B. T., Fisher, G., and Abbett, W. 2004, ApJ 610, 1148

Welsch, B. T., ApJ 638, 1101 